\title{
Boundedness on Stochastic Petri Nets
}

\author{
J. Campos, F. Plo and M. San Miguel*
}

\begin{abstract}
Stochastic Petri nets generalize the notion of queueing systems and are a useful model in performance evaluation of parallel and distributed systems. We give necessary and sufficient conditions for the boundedness of a stochastic process related to these nets.
\end{abstract}

\section{INTRODUCTION}

Petri nets are a formal tool for the modelling and validation of logical properties of parallel and distributed systems (see Peterson [7] or Silva [8] for general references). We consider a Petri net as being composed of a finite set of places numbered from 1 to $p$, and a finite set of transitions numbered from 1 to $d$. Each place can contain an integer number of 'tokens'. A $p$-tuple of null or positive integers $X=\left(X^{1}, \ldots, X^{p}\right)$ will be called a 'marking' and $X^{i}$ will be interpreted as the number of tokens in place $i$. The evolution of the marking is determined by the firing of transitions. We denote by $c_{i k}$ the number (positive, null or negative) of tokens added to place $i$ when transition $k$ is fired. The matrix $C=\left(c_{i k}\right)$,

* Research partially supported by project PA86-0028 of the Spanish Comisión Interministerial de Ciencia y Tecnología (CICYT).

1991 Mathematics Subject Classification: 60G40, 68M20, 60K25

Editorial Complutense. Madrid, 1993. 
$i=1, \ldots, p, k=1, \ldots, d$ is called the incidence matrix of the net. A transition can be fired only when there are enough resources in the system. That is, if the number of tokens in each place is not negative after the firing.

Given a firable sequence of transitions $s$, we denote by $\bar{s}$ the $d$-tuple which counts the number of times a transition $k(1 \leq k \leq d)$ occurs in $s$. The marking evolution from an initial state $X_{0}$ can be represented by $X=X_{0}$ $+C \bar{s}$. This equation is called the net firing equation and $X$ is a reachable marking from $X_{0}$.

Given a positive integer $L$, a place $k$ in the net $R$ with initial marking $X_{0}$ is said to be $L$-bounded if $X^{k} \leq L$ for each reachable marking. The net is said to be structurally bounded if for each initial $X_{0}$ and for each place $k$ there exists an integer $L$ such that $k$ is $L$-bounded.

A characterization of structural boundedness (see Brams [3]) can be given:

Theorem 1.1 [3]. Let $R$ be a Petri net with incidence matrix $C$. The three following statements are equivalent:

i) $R$ is structurally bounded.

ii) There is no $g \geq 0$ such that $C g>0$.

iii) There exists $f>0$ such that $f^{\prime} C \leq 0$.

In order to be useful as a performance evaluation tool, a timing interpretation has been added to the Petri net model, leading to stochastic Petri nets (see Molloy [5], Ajmone Marsan et al. [2], and Florin and Natkin [4]).

The purpose of this paper is to give necessary and sufficient conditions for the boundedness in mean of a stochastic process related to the evolution of the markings of a stochastic Petri net. To achieve this goal, we use tools that have been applied to the problem of optimal stopping of a process in discrete time, and particularly, the process of essential upper bounds (see Neveu [6]). The results obtained generalize the above theorem 1.1 from a stochastic point of view. 
The paper is organized as follows: in section 2, stochastic Petri nets are formally introduced. Boundedness in mean and stochastic conservation are defined; both definitions are generalized until a stopping time $T$. Section 3 includes the main result (i.e. a characterization of boundedness in mean of a positive sequence of random variables until a stopping time T) as well as two corollaries that can be seen as stochastic generalizations of theorem 1.1. Finally, an additional necessary condition for boundedness in mean of a stochastic Petri net is presented in section 4.

\section{STOCHASTIC PETRI NETS AND RELATED CONCEPTS}

2.1. Stochastic Petri nets. Stochastic Petri nets are a model that includes time in Petri nets by assigning a random variable to each transition. This variable represents the time elapsed since the transition is firable until the moment in which it actually fires. In this section we provide a stochastic process that describes the behaviour of the timed net.

Given a probability space $(\Omega, \mathscr{F}, P)$ and a family $\left(\mathscr{F}_{t}, t \geq 0\right)$ of sub- $\sigma$ fields of $\mathscr{F}$, continuous from the right, let $\left(T_{r}, r \in \mathbb{N}^{*}\right)$ be a sequence of stopping times for $\left(\mathscr{F}_{n}, t \geq 0\right)$, such that

i) $T_{r}>0$

ii) $T_{r}<T_{r+1}$ on $\left\{T_{r}<\infty\right\}$

$T_{\infty}=\lim _{n \rightarrow \infty} T_{n}=+\infty$

Let $\left(u(r), r \in \mathbb{N}^{*}\right)$ be a sequence of random variables with values in $\{1,2, \ldots, d\}$. The multivariate point process $\left(N_{\ell}\right)$

$$
N_{t}=\left(N_{t}^{1}, N_{t}^{2}, \ldots, N_{t}^{d}\right), \quad t \geq 0,
$$

where 


$$
N_{r}^{k}=\sum_{r \in \mathbb{N}}^{i} 1_{i T r<t} 1_{l u(r)=k\}} ; \quad t \geq 0 ; \quad k=1,2, \ldots, d
$$

is called firing process. If $\{u(r)=i\}$ represents the firing of transition $i$ at instant $T_{r}$, it can be interpreted that $\left(N_{r}\right)$ counts the firings of transitions until the instant $t$.

Given a stochastic vector $X_{0}=\left(X_{0}^{1}, \ldots, X_{0}^{p}\right)$, a matrix $C, p \times d$, such that $c_{j k} \in \mathbb{Z}, j=1, \ldots, p, i=1, \ldots, d$, and a firing process $\left(N_{i}, t \geq 0\right)$, a necessary condition for the firing of transition $i$ at instant $T_{r}$ is

$$
X_{T r-1}^{j}+c_{j i} \geq 0, \quad j=1, \ldots, p .
$$

We define the continuous parameter process $\left(X_{t}, t \geq 0\right)$, of $p$ components, as follows:

$$
X_{t}=X_{0}+C N_{t}, \quad \dot{t} \geq 0
$$

This process can be interpreted as the description of an evolution of markings in a stochastic Petri net, with $p$ places, $d$ transitions, incidence matrix $C$ and initial marking $X_{0}$. Condition (2.1) ensures that (2.2) is a non-negative process.

We consider in what follows the discrete parameter processes $N_{r}=N_{T r}$ and $X_{r}=X_{T r}$. The equation $X_{T r}=X_{0}+C N_{T r}$ is denoted by $X_{r}=X_{0}+C N_{r}$. This process will be called stochastic Petri net.

2.2. Boundedness in mean and stochastic conservation. In the structural analysis of a Petri net, with incidence matrix $C$ and initial marking $X_{0}$, the net is called conservative if there exist vectors $f \in\left(\mathbb{N}^{*}\right)^{p}$, such that $f^{\prime} C=0$. Similarly, for a stochastic Petri net we can search for $p$-dimensional random variables such that $f^{\prime} C\left(N_{r}-N_{r, 1}\right)=0$, or $E\left(f^{\prime} C\left(N_{r}-N_{r-1}\right)\right)$ $=0$, where $r=1,2, \ldots$ and $N_{0}=(0, \ldots, 0)$.

In the first case we try to obtain a conservative behaviour in each path and in the second, a conservative behaviour on average. 
The first case is too constraining to be useful. The second is a suitable approach but there are not enough stochastic tools for its development. Therefore, it seems convenient to follow an intermediate approach and carry out the search for a $p$-dimensional random variable $f=\left(f_{1}, \ldots f_{p}\right)$, satisfying the following conditions:

i) $f_{i} \geq 1, \quad \forall i=1, \ldots, p$

ii) $E\left(f^{\prime} C\left(N_{r}-N_{r-1}\right) \mid N_{0}, \ldots, N_{r-1}\right)=0, \quad r=1,2 \ldots$

If we consider the sequence of markings $X=\left(X_{r}\right)$, condition (2.4) can be written

ii) $E\left(f^{\prime}\left(X_{r}-X_{r-1}\right) \mid X_{0}, \ldots, X_{r-1}\right)=0, \quad r=1,2 \ldots$

We denote by $\left(X_{r}^{*}\right)$ the sequence that describes the sum of tokens in the net in each instant $T_{r}$, that is:

$$
X_{r}^{\#}=\sum_{j=1}^{p} X_{r}^{j}, \text { for each } r \geq 0 \text {. }
$$

If there exists a $p$-dimensional random variable $f$ satisfying conditions i) and ii), then the sequence

$$
f^{\prime} X=\sum_{j=1}^{p} f_{j} X_{r}^{j}
$$

is a martingale dominating $\left(X_{r}^{*}\right)$.

We must point out, however, that Doob decomposition of a supermartingale

$$
Y_{r}=M_{r}-A_{r}, \quad r \in \mathbb{N}
$$

(where $\left(M_{r}\right)$ is a martingale and $\left(A_{r}\right)$ is an increasing sequence such that $A_{0}=0$ ) ensures that the existence of a martingale dominating $\left(X_{r}^{*}\right)$ is equivalent to the existence of a supermartingale dominating $\left(X_{r}^{\#}\right)$. This 
remark as well as the previous considerations justify the following definition.

Definition 2.2.1. A stochastic Petri net $\left(X_{r}\right)$ is called stochastically conservative if there exists a supermartingale $\left(Y_{r}\right)$ dominating the sequence $\left(X_{r}^{\#}\right)$.

Let $\Lambda$ be the set of all stopping times for $\left(\mathscr{F}_{r}\right)$. The expected number of tokens at a stopping time $S \in \Lambda$ is $E\left(X_{S}^{\#}\right)$. Therefore, we can generalize the concept of boundedness (see section 1) in the following way.

Definition 2.2.2. A stochastic Petri net $\left(X_{r}\right)$ is called bounded in mean if

$$
\sup _{S \in A}\left\{E\left(X_{S}^{H}\right)\right\}<\infty .
$$

The stochastic Petri nets related to a structurally bounded Petri net are also bounded in mean. We can easily find unbounded Petri nets whose related stochastic Petri net is bounded in mean.

However, there are some unbounded stochastic Petri nets in cases in which boundedness should also be expected. For instance, the stochastic Petri net which models an ergodic $M / M / 1$ queue is not bounded in mean. This fact justifies a less exigent condition of boundedness, which we presently put forward.

2.3. Boundedness and stochastic conservation until a stopping time T. Let $L$ be a class of stopping times for $\left(\mathscr{F}_{r}\right)$. On the assumption that $X(S)$ is a $\mathscr{F}_{S}$-measurable and integrable random variable for each $S \in L$, we can give the following definition.

Definition 2.3.1. The family $\{X(S), S \in L\}$ is an $L$-supermartingale if $E\left(X(V) / F_{U}\right) \leq X(U)$, for all $U, V \in L$, where $V \geq U$. 
If the variables $X(S)$ are positive, we can drop the hypothesis of integrability over $X(S)$ in definition 2.3.1. In this case, we say that $\{X(S)$, $S \in L\}$ is a generalized $L$-supermartingale.

We are interested in the behaviour of the stochastic net until a stopping time $T$, (i.e until some condition is fulfilled or some event is observed). Therefore we take $L=\{S: S \in \Lambda, S \leq T\}$ and generalize definitions 2.2 .1 and 2.2.2 in the following way.

Definition 2.3.2. A stochastic Petri net is called stochastically conservartive until the stopping time $T$ if there exists a $L$-supermartingale dominating $\left(X_{S}^{\#}, S \leq T\right)$.

Definition 2.3.3. A stochastic Petri net is called bounded in mean until the stopping time $T$ if

$$
\sup _{S \leq T}\left\{E\left(X_{S}^{\#}\right)\right\}<\infty .
$$

Usually we take, as stopping time $T$, the first hitting time of $\left(X_{r}\right)$ in $B$, defined by

$$
T_{B}=\left\{\begin{array}{l}
\inf \left\{r \geq 0: X_{r} \in B\right\} \\
+\infty ; \text { if } X_{r} \notin B \text { for all } r \in \mathbb{N}
\end{array}\right.
$$

where $B$ is a subset of $\mathbb{N}$.

\section{A CHARACTERIZATION OF BOUNDEDNESS UNTIL A STOPPING TIME T}

In this section we present a characterization of boundedness of $\left(X_{r}\right)$, a positive sequence of random variables adapted to $\left(F_{r}\right)$, until a stopping time $T$. Two altemative stochastic generalizations of theorem 1.1 are derived as corollaries. 
Given a fixed stopping time $T$, let $L=\{S: S \in \Lambda, S \leq T\}$ and let us denote by $L_{U}$ the set of all stopping times of $L$ which are greater than or equal to the stopping time $U$. We refer to $(Y(U), U \in L)$, where

$$
Y(U)=\underset{s \in L_{U}}{\sup } \operatorname{su}\left(X_{S} \mid \mathscr{g}_{U}\right)
$$

as the family of essential upper bounds.

It can be shown (see Adell [1]) that the family $(Y(U), U \in L)$ is the smallest generalized $L$-supermartingale dominating $\left(X_{U}, U \in L\right)$.

In order to study the family $(Y(U))$ we point out that there is a correspondence between the sets $L$ and $M=\{S \wedge T: S \in \Lambda\}(S \wedge T$ stands for $\inf (S, T))$. This correspondence suggests that we take the sequence $\left(X_{r \wedge T}\right)$ instead of $\left(X_{r}\right)$ and consider the sequence $\{r \wedge T, r \in \mathbb{N}\}$ as a generating system, in the sense that

i) $r \wedge T \in L, r \in \mathbb{N}$

ii) if $S \leq T$, then $S=\sum_{r \in \mathbb{N}}(n \wedge T) 1_{\{S=r\}}$

Let $N$ be a positive integer. Given the random horizon $T$, we denote by $L^{N}$ the class of stopping times $S$ such that $S \leq N \wedge T$. The sequence ( $r \wedge T, r=0,1, \ldots, N)$ is a generating system for $L^{N}$, and, following Neveu [6] (p. 128), we can build the sequence

$$
\begin{aligned}
& Y^{N}(N \wedge T)=X_{N \wedge T} \\
& Y^{N}(r \wedge T)=\max \left(X_{r \wedge T} E\left(Y^{N}((r+1) \wedge T) \mid F_{r \wedge T}\right) \text { if } r=0,1, \ldots, N-1\right.
\end{aligned}
$$

This sequence (3.2) is the smallest generalized supermartingale dominating $\left(X_{r \wedge T}, r=0,1, \ldots, N\right)$ for each $N$ previously fixed.

For each $N$ and each $r=0,1, \ldots, N$, it holds that $Y^{N}(r \wedge T) \leq Y^{N+1}(r \wedge T)$. Thus, it is clear that the limits 


$$
\left(\lim _{N} Y^{N}(r \wedge T), r \in \mathbb{N}\right)
$$

exist. They are denoted by $\left(Y^{\infty}(r \wedge T)\right)$.

Both, the family $(Y(U), U \in L)$ of essential upper bounds and the sequence $\left(Y^{\infty}(r \wedge T)\right)$, enable us to state the following result.

Theorem 3.1. The three following statements are equivalent:

i) $\sup _{s \leq T}\left\{E\left(X_{s}\right\}<\infty\right.$

ii) There exists a supermartingale dominating $\left(X_{r \wedge T}\right)$.

(iii) There exists an L-supermartingale dominating the family of random variables $\left(X_{S}, S \in L\right)$.

Proof. [i) $\Rightarrow$ ii)] Under the hypothesis i), the random variables $Y^{\infty}(r \wedge T)$ are integrable. Indeed,

$$
E\left(Y^{\infty}(r \wedge T)\right)=E\left(\lim _{N} Y^{N}(r \wedge T)\right)=\lim _{N} E\left(Y^{N}(r \wedge T)\right)
$$

As $\left(Y^{N}(r \wedge T), r=1, \ldots, N\right)$ is a supermartingale, then

$$
\begin{aligned}
& E\left(Y^{\infty}(r \wedge T)\right) \leq \lim _{N} E\left(Y^{N}(1 \wedge T)\right)= \\
& =\lim _{N}\left(\sup _{S \leq N \wedge T} E\left(X_{S}\right)\right) \leq \sup _{S \leq T} E\left(X_{S}\right)<\infty .
\end{aligned}
$$

The supermartingale condition holds: 


$$
\left.E\left(Y^{\infty}((r+1) \wedge T) \mid \mathscr{F}_{r \wedge T}\right)=E\left(\lim _{N} Y^{N}((r+1) \wedge T)\right) \mid \mathscr{F}_{r \wedge T}\right) \leq
$$

$$
\leq \lim _{N} Y^{N}(r \wedge T)=Y^{\infty}(r \wedge T), \quad \forall r \in \mathbb{N}
$$

And

$$
Y^{\infty}(r \wedge T) \geq Y^{r}(r \wedge T)=X_{r \wedge T}, \quad \forall r \in \mathbb{N}
$$

Therefore, $\left(Y^{\infty}(r \wedge T)\right)$ is a supermartingale dominating $\left(X_{r \wedge T}\right)$.

[ii) $\Rightarrow$ iii)] Let $\left(Z_{r}\right)$ be a supermartingale dominating $\left(X_{r \wedge}\right)$. Then

$$
Z_{r} \geq Y^{N}(r \wedge T), \quad \mathbb{N}=r, r+1, \ldots
$$

and then we have

$$
Y^{\infty}(r \wedge T)=\lim _{N} Y^{N}(r \wedge T) \leq Z, \quad \forall r \in \mathbb{N}
$$

Therefore, the sequence $\left(Y^{\infty}(r \wedge T)\right)$ is the smallest supermartingale dominating $\left(X_{r \wedge T}\right)$ and $Y^{\infty}(r \wedge T)$ coincides with the essential upper bound, that is

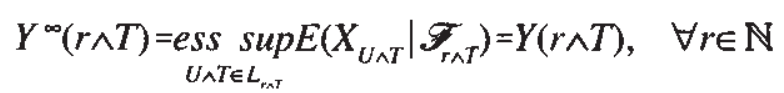

On the other hand, the family of essential upper bounds $(Y(U), U \in L)$ is the smallest generalized $L$-supermartingale dominating $\left(X_{S}, S \in L\right)$, which gives

$$
E\left(Y(S) \mid \mathscr{F}_{0}\right) \leq Y(0)=Y^{\infty}(0 \wedge T),
$$

hence, $E(Y(S)) \leq E\left(Y^{\infty}(0 \wedge T)\right)<\infty$, for all $S \in L$, and iii) holds. 
[iii) $\Rightarrow$ i)] Let $(Z(S), S \in L)$ be an $L$-supermartingale dominating $\left(X_{S}\right.$, $S \in L)$, then

$$
E\left(X_{S}\right) \leq E(Z(S)) \leq E(Z(0))<\infty \text {, for all } S \in L
$$

and

$$
\sup _{S \leq T} E\left(X_{S}\right)<\infty .
$$

Q.E.D.

From theorem 3.1 and according to definitions 2.3 .2 and 2.3.3, the next result follows.

Corollary 3.2. The following statements are equivalent: time $T$.

i) The stochastic Petri net $\left(X_{r}\right)$ is bounded in mean until the stopping

ii) The stochastic Petri net $\left(X_{r}\right)$ is stochastically conservative until the. stopping time $T$.

iii) There exists a supermartingale dominating $\left(X_{r \wedge T}^{\#}\right)$.

Corollary 3.2 allows us to give an example of a stochastic net which is bounded whereas its underlying Petri net is not. Let $\left(X_{r}\right)$ be the Petri net which models an $M / M / 1$ queue whose mean interarrival time is greater than the mean service time (i.e., an ergodic queue). The regenerative character of the sequence enables us to prove that $\left(X_{r}\right)$ is stochastically conservative until the instant of entrance in $B$, where $B$ is a set of finite cardinal from the spaces of states $E$ (for example, $B=\{0 \mid$ ). That is to say, $\left(X_{S}^{\#}, S \leq T_{B}\right)$ is an $L$-supermartingale and the net is bounded in risan until the stopping time of 'regeneration' in $B$.

If the stopping time $T$ is infinite a.s. then, according to de.initions 2.2.1 and 2.2.2, the next result follows. 
Corollary 3.3. The stochastic Petri net $\left(X_{r}\right)$ is bounded in mean if and only if it is stochastically conservartive.

Corollaries 3.2 and 3.3 constitute two stochastic generalizations of theorem 1.1.

\section{A NECESSARY CONDITION FOR BOUNDEDNESS}

The following result gives a necessary condition for boundedness in mean for stochastic Petri nets.

Theorem 4.1. Let $\left(X_{r}\right)$ be a bounded in mean stochastic Petri net. Let $m$ be a non negative integer $m$ and $\left(T_{k}\right)$ a sequence of stopping times such that $T_{k} \geq m$. If there exists a sequence $\left(Z_{r}\right)$ dominated by $\left(X_{r}\right)$ and such that $\left(E\left(X_{m}, F_{m}\right)\right)$ is a non decreasing.sequence, then

$$
\operatorname{Pr}\left\{\omega: E\left(Z_{T k}-Z_{T k-1} / \mathscr{F}_{m}\right) \geq \varepsilon, \text { i.o. }\right\}=0, \quad \forall \varepsilon>0
$$

where i.o. stands for infinitely often.

Proof. Let $\left(Z_{r}\right)$ be a sequence such that $Z_{r} \leq X_{r}$ and let $\left(T_{k}\right)$ be a sequence of stopping times such that $\left(E\left(Z_{r k} \mid \mathscr{F}_{m}\right)\right)$ is monotonous non decreasing. Let $\left(H_{r}\right)$ be the supermartingale dominating the sequence $\left(X_{r}\right)$. From the inequalities $Z_{T k} \leq X_{T k} \leq H_{T k}$ we obtain $\left(E\left(Z_{T k} \mid \mathscr{F}_{m}\right)\right) \leq H_{m}$ for each $k$.

Therefore the sequence $\left(E\left(Z_{T k} \mid F_{m}\right)\right)$ converges a.s., the sequence of its differences $\left(E\left(Z_{T k}-Z_{T k-1} \mid \mathscr{F}_{m}\right)\right)$ converge to zero a.s. and the result follows.

The next corollary can be easily inferred.

Q.E.D.

Corollary 4.2. Let $\left(X_{r}\right)$ be a stochastic Petri net. If there exists a submartingale $\left(Z_{r}\right)$ dominated by $\left(X_{r}^{*}\right)$ such that for some $\varepsilon>0$ and some $m \in \mathbb{N}$ 


$$
\operatorname{Pr}\left\{\omega: E\left(Z_{r}-Z_{r-1} / \mathscr{F}_{m}\right)>\varepsilon, \text { i.o. }\right\}>0
$$

then $\left(X_{r}\right)$ is not bounded in mean.

For stochastic Petri nets bounded in mean until the stopping time $T$ an analogous result to theorem 4.1 can be stated. In the same way, and analogous to corollary 4.2 , a necessary condition for the boundedness in mean until a stopping time $T$ can be derived.

\section{References}

[1] ADELL, J.A. (1983). Parada Optima en una Clase de Tiempos de Observación, con Aplicación al Caso Markoviano. Doctoral dissertation, Universidad del País Vasco, Bilbao, Spain.

[2] AJMONE MARSAN, M., BALBO, G. and CONTE, G. (1984). A class of generalized stochastic Petri nets for the performance evaluation of multiprocessor systems. ACM Transactions on Computer Systems, Vol. 2, No. 2: 93-122.

[3] BRAMS, G.W. (1983). Réseaux de Petri: Théorie et Pratique. T. 1. Théorie et Analyse. Paris: Masson.

[4] FLORIN, G. and NATKIN, S. (1985). Les réseaux de Petri stochastiques. Technique et Science Informatiques, Vol. 4, No. 1: 143-160.

[5] MOLLOY, M.K. (1982). Performance analysis using stochastic Petri nets. IEEE Transactions on Computers, Vol. C-31, No. 9: 913-917.

[6] NEVEU, J. (1975). Discrete Parameter Martingales. Amsterdam: NorthHolland Publishing Company.

[7] PETERSON, J.L. (1981). Petri Net Theory and the Modeling of Systems. Englewood Cliffs, NJ: Prentice Hall Inc. 
[8] SILVA, M. (1985). Las Redes de Petri en la Automática y la Informáticia. Madrid: Editorial AC.

\author{
J. Campos \\ Departamento de Ingeniería \\ Ectrica e Informática \\ Universidad de Zaragoza \\ 50015 Zaragoza \\ SPAIN \\ F. Plo-M. San Miguel \\ Departamento de Métodos Estadísticos. \\ Universidad de Zaragoza \\ 50009 Zaragoza \\ SPAIN
}

Recibido: 29 de mayo de 1991

Revisado: 4 de noviembre de 1992 